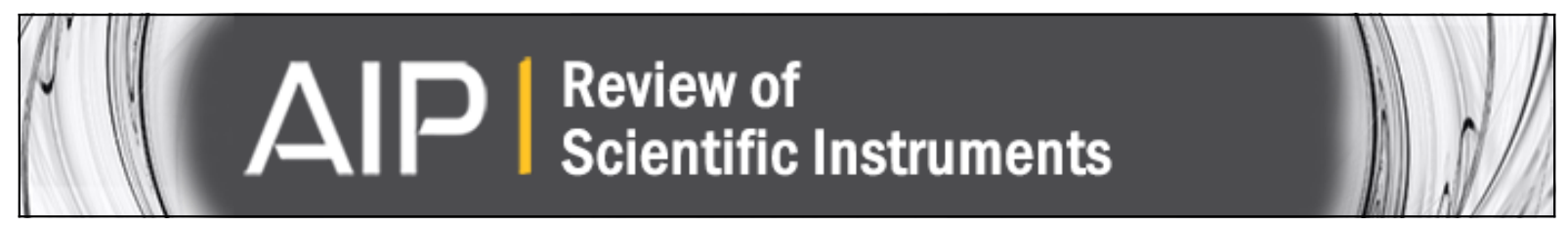

\title{
Pulsed supersonic helium beams for plasma edge diagnosis
}

T. Diez-Rojo, V. J. Herrero, I. Tanarro, F. L. Tabarés, and D. Tafalla

Citation: Review of Scientific Instruments 68, 1423 (1997); doi: 10.1063/1.1147628

View online: http://dx.doi.org/10.1063/1.1147628

View Table of Contents: http://scitation.aip.org/content/aip/journal/rsi/68/3?ver=pdfcov

Published by the AIP Publishing

Articles you may be interested in

Nonlocal effects in a bounded low-temperature plasma with fast electronsa)

Phys. Plasmas 14, 057101 (2007); 10.1063/1.2436470

Simulation of sheet-shaped lithium beam probe performance for two-dimensional edge plasma measurement Rev. Sci. Instrum. 77, 10F526 (2006); 10.1063/1.2338306

Atomic beam diagnostics for characterization of edge plasma in TJ-II stellarator

Rev. Sci. Instrum. 72, 602 (2001); 10.1063/1.1319868

Density fluctuation phenomena in the scrape-off layer and edge plasma of the Wendelstein 7-AS stellarator

Phys. Plasmas 6, 4239 (1999); 10.1063/1.873691

Measurements of edge density profiles on TdeV using the injection of laser-ablated lithium atoms

Rev. Sci. Instrum. 68, 994 (1997); 10.1063/1.1147734

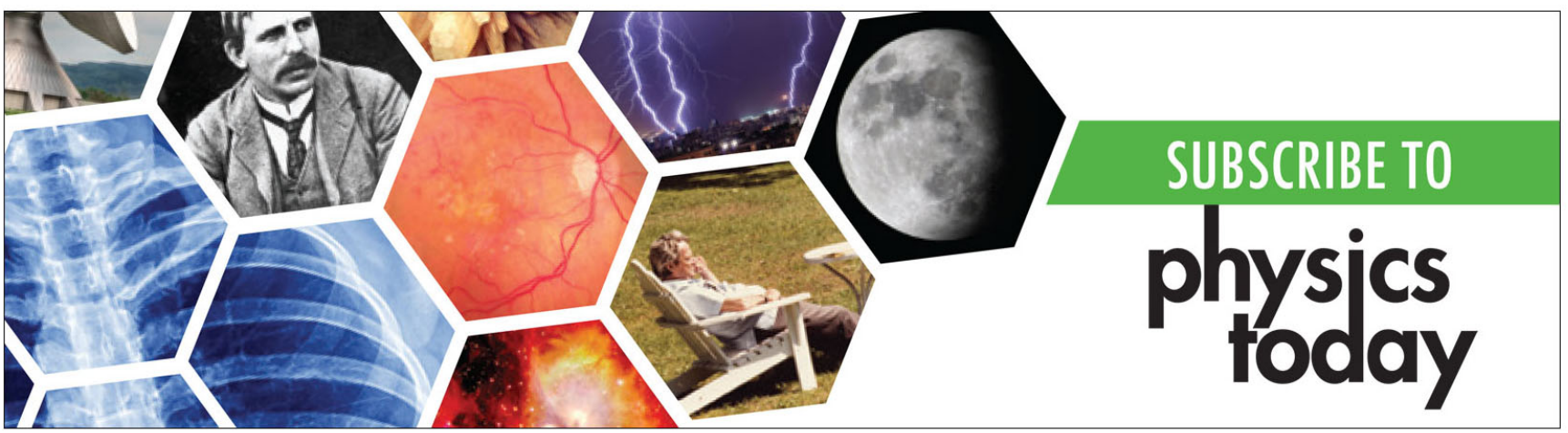




\title{
Pulsed supersonic helium beams for plasma edge diagnosis
}

\author{
T. Diez-Rojo, V. J. Herrero, and I. Tanarro \\ Instituto de Estructura de la Materia (CSIC), Serrano 123, 28006 Madrid, Spain \\ F. L. Tabarés and D. Tafalla \\ Asociación EURATOM-CIEMAT para Fusión, Avenue Complutense 22, 28040 Madrid, Spain
}

(Received 28 June 1996; accepted for publication 22 November 1996)

\begin{abstract}
An experimental setup for the production of pulsed supersonic He beams to be used for plasma edge diagnosis in fusion devices is described. A compromise between compact design, low cost, and good quality of the probe beams has been met. The main characteristics of the generated beams, such as pulse shape, absolute flux intensity, and velocity distribution, differ in general from those expected for ideal beam performance and have been determined and optimized experimentally. A first test of this He beam source at the TJ-I UP Torsatron in Madrid is also reported. (C) 1997
\end{abstract} American Institute of Physics. [S0034-6748(97)01903-5]

\section{INTRODUCTION}

Electron temperature $T_{e}(r)$ and density $n_{e}(r)$ profiles in the plasma edge of a fusion device are important parameters to describe the plasma-wall interaction and the plasma transport properties. Fluorescence spectroscopy of light atoms introduced in the plasma as effusive atomic beams and excited by electronic collisions has proved to be very suitable for the measurement of these quantities and their evolution during the discharge. ${ }^{1}$ The determination of either $T_{e}(r)$ or $n_{e}(r)$ is typically performed by studying the intensity profile of selected emission lines excited by the interaction of the beam with the plasma. The use of a low $Z$ impurity, whose excitation rate is independent of the local electron temperature, allows for the determination of the density profile. For that purpose, Li beams produced by several methods and covering a broad range of energies have been traditionally employed. ${ }^{2,3}$ A comparison between the emission intensity of lines from two injected impurities allows for the determination of the electron temperature, providing that their excitation rates show a different functionality with respect to that parameter, as is the case for the $\mathrm{Li} / \mathrm{He}$ or $\mathrm{Li} / \mathrm{C}$ combinations. Unfortunately, the different penetration properties of the injected impurities strongly restrict the spatial range in which this technique can be applied. This problem can be solved by using a single type of impurity and monitoring a set of emission lines whose ratio can be directly correlated to the plasma parameter of interest.

In that respect, the recent development of a collisional radiative model ${ }^{4}$ has allowed the use of helium atoms for the simultaneous measurements of both $n_{e}(r)$ and $T_{e}(r) .{ }^{5}$ In addition, helium atoms present the advantage of a much higher ionization potential $(25.4 \mathrm{eV})$ than lithium $(5.4 \mathrm{eV})$, and therefore a larger penetration depth. Furthermore, helium beams are easier to prepare and regulate. In order to increase the beam density, effusive beams for plasma diagnosis have usually been generated from multichannel sources. ${ }^{2}$ On the other hand, the disadvantages of this method are that effusive atomic beam diagnostics are subject to large beam divergence, offering low spatial resolution, and when employedusually with a continuous flux of gas-cause the input of an undesirably large amount of atoms into the plasma.
Supersonic beams, obtained from hydrodynamic expansions of gas into a vacuum through a nozzle, offer an interesting alternative to the effusive ones. The beams are usually extracted by collimation of the central part of the expanding gas with a skimmer. ${ }^{6}$ Until now, they have had very successful applications in other fields of physics research; they provide a higher mean translational velocity of the atoms than the thermal ones (higher penetration depths in the plasma) with a remarkably narrower velocity spread (better temporal resolution), and a lower angular divergence determined by the geometry of the nozzle and the skimmer (better spatial resolution). These features result in easier modeling of beam-plasma interaction.

The properties of supersonic beams cannot be predicted as easily and accurately as those of effusive beams. By neglecting viscosity and heat conduction effects (i.e., by assuming that the expansion is isentropic), a series of models and empirical correlations have been developed in an attempt to estimate the terminal flow velocity, the velocity distribution in the direction of propagation of the beam, and the total particle flow rate through the nozzle (see for instance Ref. 6 and references therein). Nevertheless, deviations of real systems from these expected values, which can be particularly significant for the flux intensity beyond the skimmer, are strongly dependent on the particular experimental setup; consequently, they should be determined experimentally. Such deviations are caused mainly by nonadiabatic effects during the expansion, perturbations of the molecular beam by the skimmer, and scattering of the beam particles by background gas molecules.

Due to the high source pressure used in hydrodynamic expansions, the production of continuous supersonic beams requires, in general, a relatively large expansion chamber and large vacuum pumps, and these requirements increase the cost of the diagnostic system and the difficulty for practical access to the plasma device. Taking into account the pulsed nature of plasma generation in fusion devices, pulsed atomic beams can be used to circumvent these inconveniences. The generation of pulsed supersonic beams can be done in smaller chambers and with much smaller pumping speeds. In addition, the pulsed operation causes a much lower total influx of the probe species to the plasma device. However, an 


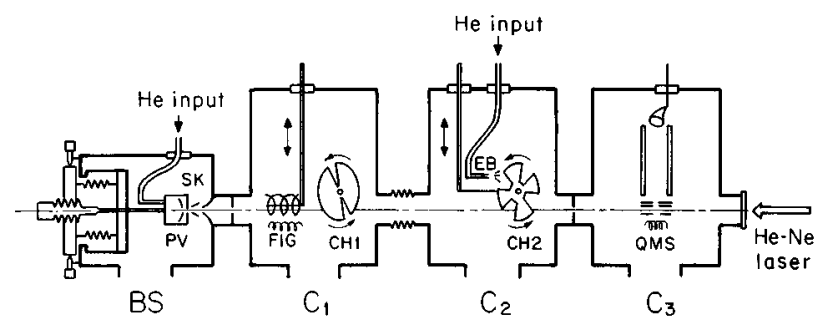

FIG. 1. Experimental setup for the characterization of the He supersonic pulsed beam. BS is the beam source chamber used for plasma diagnosis, $\mathrm{PV}$ the pulsed valve, and $\mathrm{SK}$, the skimmer. $\mathrm{C}_{1}, \mathrm{C}_{2}, \mathrm{C}_{3}$ are the vacuum chambers for beam analysis; FIG, the fast ionization gauge; $\mathrm{CH} 1$, the chopper for time of flight measurements; $\mathrm{EB}$, the effusive He beam; $\mathrm{CH} 2$, the chopper for the effusive beam modulation, and QMS, the quadrupole mass spectrometer.

excessive reduction of the expansion volume results in a significant deterioration of the beam properties, and an equilibrium must be found between the small of the source and the desirable characteristics of the supersonic beams produced.

In this work a pulsed supersonic beam source of $\mathrm{He}$, developed for plasma edge diagnostics and installed at the TJ-IU Torsatron in Madrid, is described. A compromise has been made between the compact design and good quality of the probe beams. The fundamental beam properties such as pulse shape, absolute flux intensity, velocity distribution, and beam divergence have been determined experimentally and are compared to the expected values for ideal beam performance. The first results of the application of the beam source to plasma diagnosis are also shown.

\section{ATOMIC BEAM SOURCE}

The atomic beam source consists of a small expansion chamber, which contains a pulsed valve and a skimmer. This chamber can be connected either to the TJ-1U Torsatron or to the system for beam analysis (Fig. 1) through a CF-35 flange. The supersonic beam is formed by expanding helium through the pulsed valve into the vacuum chamber and extracting its central part by means of the skimmer. The skimmer is a commercial one (Beam Dynamics), with a circular diaphragm $0.5 \mathrm{~mm}$ in diameter, a parabolic profile, and a very thin wall near the diaphragm $(100 \mu \mathrm{m})$; it was selected in order to minimize skimmer perturbations on the beam. The vacuum chamber was built with low magnetic permeability stainless steel (SS 316L). It has several CF-35 flanges for the manometers and for electric and gas connections and a CF-100 flange for vacuum pumping. It is rather small in order to allow its coupling to the TJ-1U Torsatron of Madrid. Its internal volume is $V \approx 3 \ell$. It is pumped by a turbomolecular pump with an extracting capacity for He of $Z=240$ $\ell / \mathrm{s}$, backed by a rotary pump, so the pumping time constant is $V / Z \approx 12 \mathrm{~ms}$. The pulsed valve is a commercial one (Laser Technics LPV), with an electro-mechanical opening system based on a bimorph piezoelectric crystal which moves the shutting stem, activated in a continuous or pulsed way by means of an electrical signal. This valve was selected to expand the He gas, instead of the more usual and compact solenoid driven valves, in order to avoid magnetic interactions near the plasma. It is mounted on a movable base that can be displaced under vacuum $\approx \pm 5 \mathrm{~mm}$ in both directions perpendicular to the beam axis by means of a flexible bellow, in order to allow the alignment of the molecular beams, and $\approx 20 \mathrm{~mm}$ in the axial direction by means of a micrometer screw, in order to regulate the nozzle-skimmer distance. Gas is supplied to the valve through a thin flexible tube.

The nozzle orifice of the valve is contained in an adjustable piece with a screw thread. It should be screwed carefully in the front part of the valve for proper adjustment of the "closed"' position. In this work a circular nozzle of 0.3 $\mathrm{mm}$ nominal diameter is used. The effective aperture diameter of pulsed valves can be regulated by the amplitude of the electrical signal, but the maximum opening is often smaller than the nominal one. ${ }^{7,8}$

The effective diameter was estimated from measurements of the gas flow and pulse shape performed in a separate expansion chamber. The procedure is described in detail in Ref. 8. Gas pulses of $0.5,1$, and $2 \mathrm{~ms}$ at maximum allowable voltage $(150 \mathrm{~V})$ were recorded at the exit of the valve (at a distance of $\approx 2 \mathrm{~cm}$ ) with a fast ionization gauge (FIG) for stagnation pressures up to 2 bar. All of them had a nearly rectangular temporal shape, with a well defined plateau and a rapid rise and descent slope. It should be noted that proper performance greatly depends of the adequate adjustment of the nozzle to the valve, as mentioned before, and a poor adjustment can result in the appearance of aperture oscillations during the pulse. The effective diameter corresponding to the pulse plateau is nearly constant, independently of pulse duration and stagnation pressure, and in our case its value is $0.25 \mathrm{~mm}( \pm 10 \%)$. With this value, the effective opening area is $30 \%$ smaller than the nominal one. Between the beginning of the electrical driving pulse and the actual gas pulse, a systematic delay of $150 \mu$ s was observed.

The fast ionization gauge used for these measurements is similar to an open Bayard-Alpert detector and was built in our laboratory according to the design of Gentry and Giese ${ }^{9}$ with slight modifications. In our case it was designed to eventually be used inside the TJ-1U Torsatron for atomic beam alignment, and the amplifier and all the electronic systems are placed outside the vacuum chamber for a higher lifetime of the device and for noise shielding. Under these conditions the time constant of the detector is $5 \mu \mathrm{s}$. This detector is mounted on a sliding axis in order to withdraw it from the beam path and to facilitate the alignment procedure. The detector has a small stainless steel screen at one side, with a series of small holes parallel to its incandescent filament, designed for optical alignment of the fluorescence optics prior to plasma generation.

\section{ANALYSIS SYSTEM AND PULSED VALVE CHARACTERIZATION}

In general it is not possible to predict the properties of a supersonic beam accurately and they must be obtained from measurements. In order to determine the characteristics of the helium beam to be used for plasma edge diagnostics, our atomic beam source (i.e., the chamber with the nozzleskimmer assembly) was connected to an experimental setup designed for analysis of the beams. This setup is very similar to one used previously in our laboratory. ${ }^{8}$ In the present experiments it was formed by three vacuum chambers placed in 
series (Fig. 1); the first one provided the movable fast ionization gauge described before and a chopper for time of flight facility; the second one provided a modulated effusive He beam used as a standard for the calibration of absolute intensities; and the third one communicated with the second chamber through a diaphragm (5 $\mathrm{mm}$ diameter) with a quadrupole mass spectrometer (QMS). The center of the electron impact ionizer of the QMS was placed at a distance $92 \mathrm{~cm}$ from the skimmer and $\mathrm{He}$ ions were detected by means of a secondary electron multiplier, feeding a fast current amplifier. The whole analysis system was aligned with the atomic beam source by means of a helium-neon laser. The whole system was differentially pumped. Background pressures in the $10^{-7}$ mbar range at the mass spectrometer chamber and below $5 \times 10^{-6}$ mbar in the others were maintained during the supersonic beam experiments. Under these conditions, beam attenuation effects after the skimmer can be neglected.

\section{PULSED-BEAM SHAPE AND DIVERGENCE}

Given the small volume of the expansion chamber and its pumping time constant (see Sec. III) there is a significant buildup of background pressure during the production of a typical gas pulse. As a result, the absolute intensities and the temporal profiles of the pulsed supersonic beams can be significantly affected by attenuation through the gas in the expansion chamber. In addition, the presence of the skimmer and of its supporting wall can severely perturb the ideal centerline intensity of the atomic beams. ${ }^{6,10,11}$

The source position was optimized by varying the nozzle-skimmer distance in order to obtain a maximum helium signal in the mass spectrometer. The best distance was found to be $1.5 \mathrm{~cm}$ for source stagnation pressures up to 2 bar. Smaller distances resulted in a decrease in the beam intensity caused by skimmer interference. For distances shorter than $0.5 \mathrm{~cm}$ this effect leads to the practical destruction of the beam. On the other hand, a (Beer-Lambert) attenuation of the beam due to scattering with residual gas molecules is observed for distances larger than $2 \mathrm{~cm}$. During a pulse, the background pressure increases rapidly with time after opening of the valve and is largest at the end; thus the attenuation effects are more marked for the longer pulses and for the strongest expansions. The nearly square gas pulse shape measured at the exit of the valve will thus be modified in the pulsed beam after the skimmer due to the effects just commented on and the recording of the actual beam profiles is necessary for a correct interpretation of the plasma data.

The temporal profiles of the pulsed beams were measured with the quadrupole mass spectrometer, separated by two differential pumping stages from the expansion chamber (see Fig. 1), as well as with the fast ionization gauge (FIG) mentioned above. The FIG was placed at a distance of $30 \mathrm{~cm}$ from the nozzle, which is the approximate distance between the He beam source and the plasma edge at TJ-1U. These measurements allowed us to also check this movable detector for future alignment of the beam with the collection optics in the fusion device. For a given pulse both detectors gave nearly identical time profiles. A typical example of one of these pulses is shown in Fig. 2(a). The intensity of the He beam is not constant during the pulse, but decays from be-
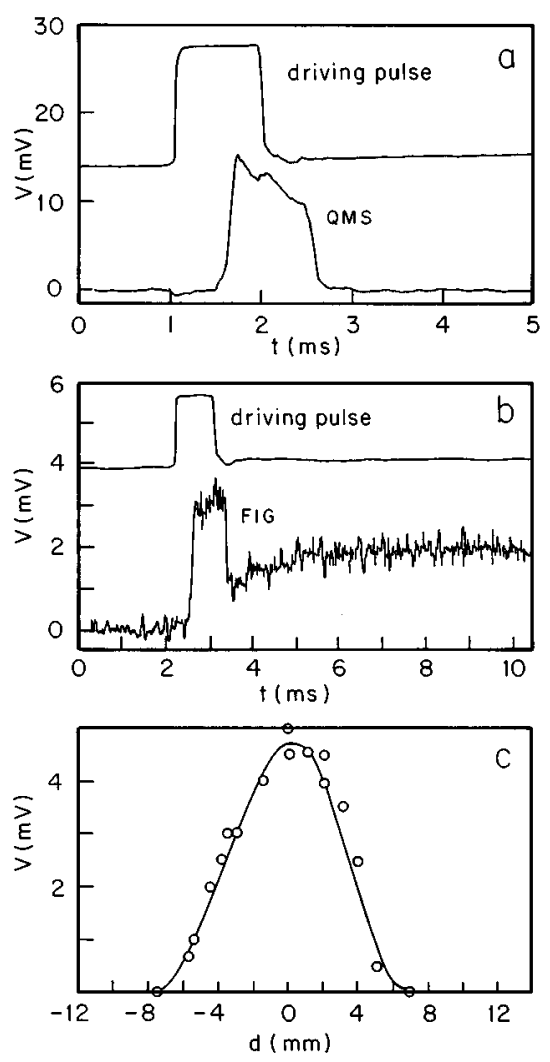

FIG. 2. (a) The temporal profile of a $1 \mathrm{~ms}, 1$ bar, He pulsed beam, recorded with the QMS, separated by two differential pumping stages from the expansion chamber. (b) The temporal profile of the same pulsed beam, recorded with the FIG in the vacuum chamber next to the expansion one. (c) The beam width at a distance of $30 \mathrm{~cm}$ from the nozzle, measured with the fast ionization gauje.

ginning to end. Such profiles have been recorded for the conditions relevant to the present diagnostics, i.e., for values of the stagnation pressure up to 2 bar and for pulses between 0.5 and $2 \mathrm{~ms}$. The signal corresponding to the supersonic beam recorded at the FIG is followed by a weaker and longer (tens of milliseconds) tail [see Fig. 2(b)] caused by the background gas in the expansion chamber diffusing through the skimmer. This flow of residual He has a large divergence and is not seen, as expected, by the mass spectrometer. Although the total amount of He introduced into the plasma by this method is remarkably lower than that introduced by continuous effusive beams, the introduction of a small differential pumping stage between the expansion chamber and the TJ-1U Torsatron would even greater decrease this secondary helium input to the plasma. Alternatively, the background pressure of the expansion chamber would be decreased by increasing its volume.

The angular divergence of the supersonic beam can be assumed, in principle, to be determined by geometry. For a nozzle skimmer separation of $1.5 \mathrm{~cm}$ and a skimmer diameter of $0.5 \mathrm{~mm}$ the divergence should be approximately $2^{\circ}$, notably smaller than the one reported for diagnostics based on effusive beams. At a distance of $30 \mathrm{~cm}$ from the nozzle the beam full width at half-maximum (FWHM) measured by shifting the FIG perpendicularly to the beam axis, is approximately $1 \mathrm{~cm}$ [see Fig. 2(c)], which is of the order 

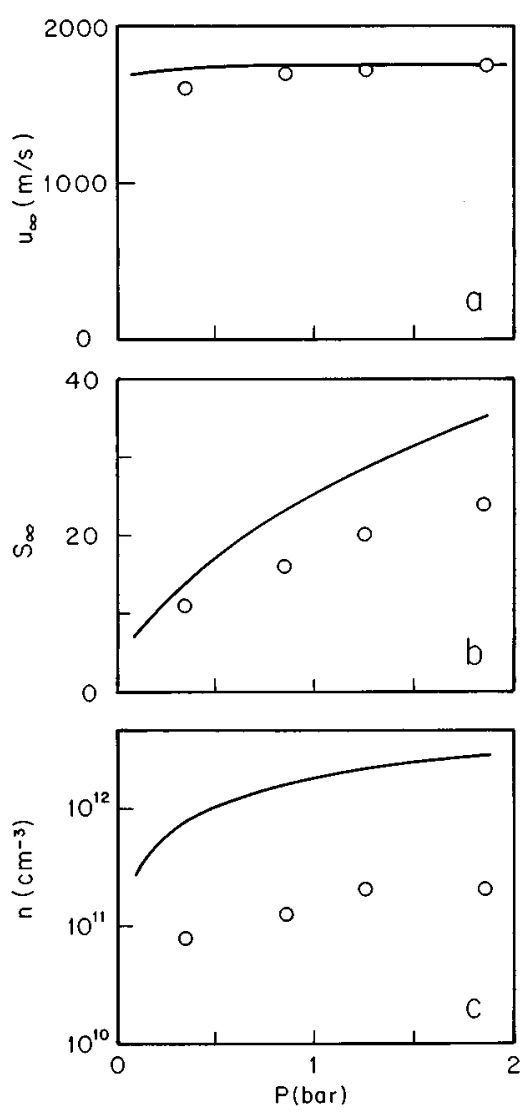

FIG. 3. (a) Terminal flow velocities of the He beam as a function of the source pressure. Open circles-experimental data. Continuous linetheoretical predictions in the isentropic approximation [Eq. (1)]. (b) Experimental (open circles) and theoretical (continuous line) terminal speed ratios from Eq. (2). (c) Experimental (open circles) and theoretical (continuous line) density values of the $\mathrm{He}$ beam at a distance of $30 \mathrm{~cm}$ from the nozzle orifice, considering the screening effect caused by the skimmer [Eq. (4)].

expected [in fact the actual beam width is somewhat smaller, since the angular distribution depicted in Fig. 2(c) has not been deconvoluted with the finite size of the ionization region of the FIG]. In order to reduce this width even more, a collimator may be placed after the skimmer.

\section{VELOCITY DISTRIBUTIONS}

The velocity distributions of the pulsed supersonic He beams were determined from time of flight (TOF) measurements in a way entirely analogous to that described in Ref. 8. In the present work the flight length between the chopper blades and the ionizer of the QMS was $60 \mathrm{~cm}$ and the gate function of the chopper, of approximately Gaussian shape, had a FWHM of $23 \mu$ s. The analysis and deconvolution of data have been done according to standard procedures. ${ }^{12}$ For the velocity distribution in the direction of propagation of the beam we have assumed the usual "drifting Maxwellian", shape, ${ }^{6}$ i.e., a Maxwellian distribution characterized by a temperature $T$, traveling with the flow velocity $u$. As a result of the expansion, a substantial narrowing (a cooling in terms of $T$ ) occurs. A common way of describing the spread in the velocities of the beam is the use of the "speed ratio" $S$, defined as the quotient between the flow velocity $u$ and the most probable thermal velocity $\nu=\sqrt{2 k T / m}$. In Figs. 3(a) and 3(b) we have represented the terminal flow velocities and speed ratios obtained from our time of flight (TOF) measurements.

The theoretical terminal flow velocities, $u_{\infty}$, in the isentropic approximation, are given by ${ }^{6}$

$$
u_{\infty}=M_{\infty} \sqrt{\frac{\gamma k T_{0}}{m}}\left(1+\frac{\gamma-1}{2} M_{\infty}^{2}\right)^{-1 / 2},
$$

where $\gamma$ is the ratio of specific heats, $k$ is Boltzmann's constant, $T_{0}$ the source temperature, $m$ the molecular mass, and $M_{\infty}$ the Mach number at the end of the expansion, which is related to the terminal speed ratio $S_{\infty}$ through $M_{\infty}$ $=\sqrt{2 / \gamma} S_{\infty}$. In most cases the measured terminal flow velocities are quite close to the limiting value, $u_{m}$ $=\sqrt{2 k T_{0} \gamma /(\gamma-1) m}$, given by the conservation of energy. ${ }^{6,13}$ A series of models and semiempirical correlations have been developed in an attempt to relate the terminal speed ratio and thus the approximate location of the end of the expansion (i.e., the place where collisions cease ${ }^{6}$ ), to the source properties. In Fig. 3(b) we also compare the results of our measurements to the values given by the model of Beijerinck and Verster: ${ }^{10}$

$$
S_{\infty}=A K n_{0}^{B},
$$

where $K n_{0}$ is the source Knudsen number and the parameters $A$ and $B$ take the values 0.527 and 0.545 , respectively, for monoatomic gases. Using the previous expressions for $u_{\infty}$ and $S_{\infty}$ one obtains the theoretical curves represented in Figs. 3(a) and 3(b).

Except for the weakest expansion considered (350 mbar source pressure), the terminal flow velocities coincide within experimental error with those given by expression (1). In the case of the 350 mbar expansion, the terminal flow velocity corresponds to approximately $92 \%$ of the theoretical values. The measured speed ratios are in all cases lower than the calculated ones, nevertheless, they are always larger than 10 and correspond thus to beams with a much narrower kinetic energy distribution than those obtained from effusive multichannel sources. ${ }^{1}$

\section{CALIBRATION OF ABSOLUTE INTENSITIES}

For the determination of the supersonic beam intensities we have used the quadrupole mass spectrometer. In order to get the absolute intensity of a beam from the corresponding QMS signal, it is necessary to calibrate the detector and this calibration must be made in situ, since the detection efficiency is different for each substance and is subject to change over a relatively short period of time, due to contamination or to drifts in the electrical conditions. As a system for in situ calibration we have used an effusive He beam generated from a movable source.

The effusive source used for the calibration of our QMS consists of a circular hole of $250 \pm 5 \mu \mathrm{m}$ diameter, burnt on a $0.1 \mathrm{~mm}$ width stainless steel foil by using the focused output of an $\mathrm{Ar}^{+}$laser. The shape and size of the hole were checked by means of an optical microscope, provided with a rectangular grid of $10 \mu \mathrm{m}$ resolution. A circular piece of the stainless steel foil was cut around the orifice and brazed with a 
silver alloy to the end of a $4 \mathrm{~mm}$ internal diameter copper tube, which is installed, together with a chopper for the modulation of the outgoing beams, in a brass housing. It is mounted in the vacuum chamber placed next to that of the QMS (see Fig. 1), on a sliding and rotary, vacuum feedthrough. Additional feedthroughs are provided for gas inlet and for the electrical connections of the chopper. The connection between the beam source and the gas feedthrough is made by means of a flexible steel tubing. The pressures in the effusive source were maintained below $1 \mathrm{mbar}$ and were monitored continuously with a "pirani" gauge, mounted close to the outer side of the vacuum flange, previously calibrated for He with a $\mathrm{Hg}$ compression manometer. The gas flow to the source was controlled by means of a pressure regulator and a stainless steel fine metering valve.

In the aligned position, the distance between the source orifice and the center of the ionization region of the QMS is $23 \mathrm{~cm}$. The effusive beam chamber is communicated with that of the QMS by means of a circular collimator $5 \mathrm{~mm}$ in diameter placed $7 \mathrm{~cm}$ from the source. During operation, the pressure in the effusive source chamber was kept below $2 \times 10^{-5}$ mbar and that of the QMS in the $10^{-7}$ range, so that the attenuation of the effusive beam was negligible. The defining aperture for the size of both effusive and supersonic beams in the ionizer was the same, namely a circular collimator of $3.1 \mathrm{~mm}$ diameter belonging to the ionizer assembly and placed $21 \mathrm{~mm}$ at the center of the ionization region. Under the conditions just described, and given the very small solid angle subtended by the detector, the properties of the calibrating beams generated by our experimental setup are not expected to differ appreciably from the theoretical centerline properties of an ideal effusive beam. ${ }^{14}$ From the calculated atomic density at the ionizer we get the calibration factor for the QMS.

After the calibration of the QMS, the effusive source is removed from the beam path and the densities of the supersonic beams are recorded. In order to check the stability of the QMS, the calibration is performed both before and after the measurement of a series of supersonic beam densities. Once the supersonic beam density at the QMS is known, it can be estimated at any other point between the QMS and the skimmer, just by considering that the density falls as the square of the distance to the source. In Fig. 3(c) we have represented the He supersonic beam densities at $30 \mathrm{~cm}$ from the nozzle orifice, which is the approximate distance between the beam source and the plasma edge at the TJ-1U Torsatron. As can be seen the densities obtained with the present setup reach values up to $2 \times 10^{11} \mathrm{~cm}^{-3}$, two times smaller than those reported for the multichannel effusive beam diagnosis at TEXTOR. ${ }^{2}$

In a simple picture one may assume that the supersonic expansion is sharply divided into a continuum flow (where collisions still take place) and a free molecular flow (without collisions) by a "quitting surface." The position of the quitting surface can be estimated, for instance, from Eq. (2) and from the evolution of the Mach number with source distance. $^{6}$ According to Beijerinck and Verster ${ }^{10}$ the axial intensity of a free jet expanding from an orifice is given by

$$
I_{0}\left(\mathrm{sr}^{-1} \cdot \mathrm{s}^{-1}\right)=\frac{\kappa(\gamma)}{\pi} \dot{N},
$$

where $\kappa(\gamma)$ is the "peaking" factor (approximately 2 for $\gamma=5 / 3)$ and $\dot{N}$ is the nozzle flow rate..$^{6,10,13}$ As mentioned above, the centerline intensity of a skimmed supersonic beam is always smaller than that given by (3) due to skimmer interaction and background gas scattering. A primary effect of the skimmer interaction is basically of geometrical nature and corresponds to the effective screening caused by the skimmer orifice on the atoms leaving the quitting surface with an average velocity perpendicular to the jet streamlines given by $S_{\infty}$. Considering this screening effect, the approximate centerline intensity of the supersonic beam can be written ${ }^{6}$

$$
\frac{I}{I_{0}} \cong 1-\exp \left[-S_{\infty}^{2}\left(\frac{r}{x_{q}}\right)^{2}\left(\frac{x_{d}}{x_{d}-x_{s}}\right)^{2}\right],
$$

where $r$ is the skimmer radius, and $x_{q}, x_{s}$, and $x_{d}$ are the distances from the source origin to the quitting surface, the skimmer, and the detector, respectively. Expression (4) takes into account the "Mach number focusing" 15 and is similar to that obtained in the "narrow virtual source" model of Beijerink and Verster. ${ }^{9}$ In Fig. 3(c) we have represented the density values calculated for our conditions with Eq. (4) and with the theoretical values from Fig. 3(b). As can be seen, the calculated values are much larger than the measured ones. The difference between measurements and calculations is due to the attenuation by collisions with atoms reflected from the inner surfaces of the vacuum chamber and with residual gas atoms. These effects, which are particularly severe in our case, are very difficult to estimate theoretically, ${ }^{11}$ but could be minimized with an increase in expansion volume and pumping speed, resulting in a smaller background pressure in the expansion chamber. In general, peak background pressures below $10^{-3}$ mbar and nozzle-skimmer distances larger than 2-4 cm lead to a significant increase in beam intensities. In fact, in experiments carried out with the same pulsed valve, but with a $50 \ell$ chamber and a $2000 \ell / \mathrm{s}$ diffusion pump, we were able to obtain beam intensities five to seven times larger than those reported here; however, both the size of the chamber and the presence of potentially contaminating diffusion pump oil preclude the use of this setup in the TJ-IU Torsatron. Even in fusion devices where space limitations are less important, eventual advantages of a higher beam intensity should be traded off with the increase in cost associated with the use of big vacuum chambers and large turbomolecular pumps.

\section{TESTING OF THE SOURCE IN THE TJ-I UP TORSATRON}

The beam source was installed in the TJ-I Upgrade for testing. ${ }^{16}$ The device ${ }^{17}$ is a $l=1, m=6$ torsatron with major radius $R=0.6 \mathrm{~m}$ and minor radius $a=0.10 \mathrm{~m}$. The magnetic field at the axis is $0.5-0.7 \mathrm{~T}$. Electron cyclotron heating $(\mathrm{ECH})$ plasmas are generated and heated using a $37.5 \mathrm{GHz}$ gyrotron ( $X$-mode, second harmonic, $P=90-250 \mathrm{~kW}$, pulse length $20 \mathrm{~ms})$. Line average electron densities of about $(0.2-0.5) \times 10^{13} \mathrm{~cm}^{-3}$ and electron temperatures in the range 


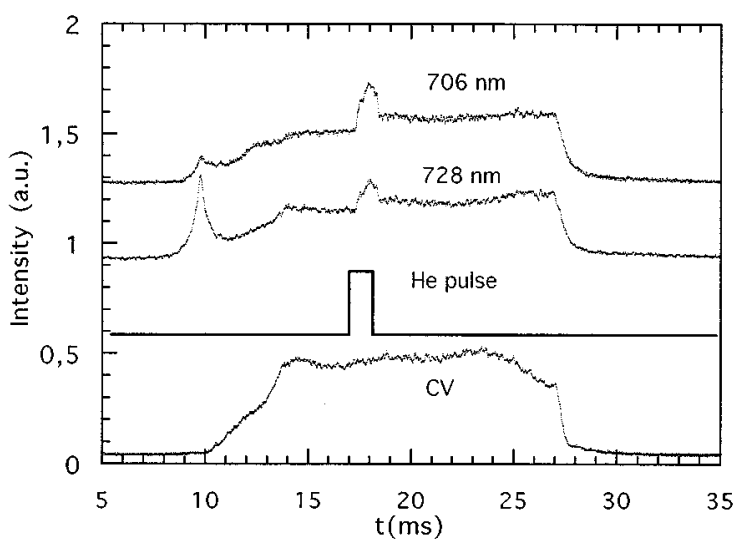

FIG. 4. Evolution of some emission line intensities during the plasma shot. From top to bottom: He lines at 706 and $728 \mathrm{~nm}$ at normalized radius $\rho=0.45$, beam pulse reference and $C V$ line. Data uncorrected for calibration factors.

of $100-200 \mathrm{eV}$, are typically obtained. Langmuir probe measurements of plasma edge parameters near the last closed magnetic surface typically yield values of $T_{e}(a) \approx 10-15 \mathrm{eV}$ and $n_{e}(a) \approx(0.5-1) \times 10^{12} \mathrm{~cm}^{-3}$. Access to the plasma is provided through 18, 250 ISO $\mathrm{K}$ flanges. The beam source is coupled to an equatorial flange through a $35 \mathrm{CF}$ flange. A bottom window, which is displaced $20^{\circ}$ in the toroidal direction with respect to the He beam input, was used for detection of the beam emission. This allowed an almost tangential observation of the plasma periphery. A set of two mirrors was used to collect the plasma light which was then imaged through a lens $(f=25 \mathrm{~cm}, \Phi=50 \mathrm{~mm})$ onto two detectors. The latter consisted of an interference filter coupled to a photomultiplier (PMT, Hamamatsu R3896). A 50\% beamsplitter was used for the simultaneous recording of the two He emission lines monitored for temperature measurements, i.e., at 706.5 and $728.1 \mathrm{~nm} .^{5}$ A spatial resolution of about 4 $\mathrm{mm}$ parallel to the He beam is reached by placing a collimator in the entrance of the PMT. The full beam width determines the poloidal and toroidal resolution. The sensitivity of the whole system was calibrated with the ratio of He lines in the He glow discharge used as routinary wall conditioning procedure in the TJ-I U torsatron. Figure 4 shows a typical example of the $\mathrm{He}$ emission line intensities during the plasma shot, lasting from $t=9$ to $t=28 \mathrm{~ms}$. As can be seen, an almost instantaneous rise of these signals follows the injection of the beam pulse in the plasma, providing excellent signal-background discrimination. The delayed influx of He, originated by the pressure rise in the source chamber during the beam formation, produces a residual signal after the beam termination. The ratio of this signal to that from the beam was found to depend on the radial position being monitored, as expected from the different spatial regions where such emissions are produced. For the case shown in the figure, corresponding to normalized radius $r / a=0.45$, a very low ratio can be observed. Within the uncertainties of the application of the collisional-radiative model to the TI-IU conditions, ${ }^{16}$ the electron temperatures inferred from the intensities ratios range from $10 \mathrm{eV}(r=a)$ to $40-50 \mathrm{eV}(r / a$ $=0.45)$. A typical value of $T_{e}=10-15 \mathrm{eV}$ is obtained by the Langmuir probes at the plasma periphery $(r \approx a)$. No comparison with other diagnostics was available for inner regions. Also shown in the figure is the evolution of an intrinsic impurity (carbon) closely related to the total plasma radiation. No effect of the He injection is observed on that emission line, in agreement with the nonperturbative characteristics of the beam expected from the associated low $\mathrm{He}$ influx.

\section{ACKNOWLEDGMENTS}

The authors are indebted to J. Rodríguez for the construction of the fast ionization gauge used in this work. T.D.-R. acknowledges the financial support through a F.P.I. fellowship of the Ministry of Education of Spain. This work was partially financed by the DGICYT of Spain under Grant No. PB94-0128.

${ }^{1}$ E. Hintz and B. Schweer, Plasma Phys. Control. Fusion 37, A87 (1995).

${ }^{2}$ A. Pospieszczyk, F. Aumayr, H. L. Bay, E. Hintz, P. Leismann, Y. T. Lie,

G. G. Ross, D. Rusbüldt, R. P. Schorn, B. Schweer, and H. Winter, J. Nucl. Mater. 574, 162 (1989).

${ }^{3}$ R. P. Schorn, E. Hintz, S. Musso, and B. Schweer, Rev. Sci. Instrum. 60, 3275 (1989).

${ }^{4}$ B. Brosda and B. Pohlmeyer, He-Beam Internal Report Ruhr-Universität Bochum (1990).

${ }^{5}$ B. Schweer, G. Mank, A. Pospieszczyk, B. Brosda, and B. Pohlmeyer, J. Nucl. Mater. 174, 196 (1992).

${ }^{6}$ D. R. Miller, in Atomic and Molecular Beam Methods, edited by G. Scoles (Oxford University Press, New York, 1988), Vol. 1, Chap. 2.

${ }^{7}$ P. Andresen, M. Faubel, D. Haeusler, G. Kraft, H. W. Luelf, and J. G. Skofronick, Rev. Sci. Instrum. 56, 2038 (1985).

${ }^{8}$ L. Abad, D. Bermejo, V. J. Herrero, J. Santos, and I. Tanarro, Rev. Sci. Instrum. 66, 3826 (1995).

${ }^{9}$ W. R. Gentry and C. F. Giese, Rev. Sci. Instrum. 49, 595 (1978).

${ }^{10}$ H. C. W. Beijerinck and N. F. Verster, Physica C 111, 327 (1981).

${ }^{11}$ M. J. Verheijen, H. C. W. Beijerinck, W. A. Renes, and N. F. Verster, Chem. Phys. 85, 63 (1984).

${ }^{12}$ D. Auerbach, in Atomic and Molecular Beam Methods, edited by G. Scoles (Oxford University Press, New York, 1988), Vol. 1, p. 362.

${ }^{13}$ M. Zucrow and J. Hoffman, in Gas Dynamics (Wiley, New York, 1976), Vols. I and II.

${ }^{14}$ H. Pauli, in Atomic and Molecular Beam Methods, edited by G. Scoles (Oxford University Press, New York, 1988), Vol. 1, Chap. 3.

${ }^{15}$ P. K. Sharma, E. L. Knut, and W. S. Young, J. Chem. Phys. 64, 4345 (1976)

${ }^{16}$ F. L. Tabarés, D. Tafalla, V. J. Herrero, and I. Tanarro, J. Nucl. Matter. (in press).

${ }^{17}$ E. Ascasibar et al., Trans. Fusion Technol. 27, 198 (1995). 